\title{
High-Performance Perovskite Light-Emitting Diode with Enhanced Operational Stability Using Lithium Halide Passivation
}

Tian Wu, Junnan Li, Yatao Zou, Hao Xu, Kaichuan Wen, Shanshan Wan, Sai Bai, Tao Song, John A. McLeod, Steffen Duhm, Feng Gao and Baoquan Sun

The self-archived postprint version of this journal article is available at Linköping University Institutional Repository (DiVA):

http://urn.kb.se/resolve?urn=urn:nbn:se:liu:diva-163620

N.B.: When citing this work, cite the original publication.

Wu, T., Li, J., Zou, Y., Xu, H., Wen, K., Wan, S., Bai, S., Song, T., McLeod, J. A., Duhm, S., Gao, F., Sun, B., (2020), High-Performance Perovskite Light-Emitting Diode with Enhanced Operational Stability Using Lithium Halide Passivation, Angewandte Chemie International Edition.

https://doi.org/10.1002/anie.201914000

Original publication available at:

https://doi.org/10.1002/anie.201914000

Copyright: Wiley (12 months)

http://eu.wiley.com/WileyCDA/ 


\section{High-Performance Perovskite Light-Emitting Diode with Enhanced Operational Stability Using Lithium Halide Passivation}

Tian $\mathrm{Wu}^{\ddagger 1}$, Junnan $\mathrm{Li}^{\ddagger 1}$, Yatao Zou ${ }^{\ddagger 1,2}$, Hao Xu${ }^{1}$, Kaichuan $\mathrm{Wen}^{3}$, Shanshan Wan ${ }^{1}$, Sai Bai $^{* 2}$, Tao Song ${ }^{* 1}$, John A. McLeod ${ }^{* 1}$, Steffen Duhm ${ }^{1}$, Feng Gao ${ }^{2}$, Baoquan Sun ${ }^{* 1}$

${ }^{1}$ Jiangsu Key Laboratory for Carbon-Based Functional Materials \& Devices, Institute of Functional Nano \& Soft Materials (FUNSOM), Soochow University, 199 Ren'ai Road, Suzhou 215123, People's Republic of China

${ }^{2}$ Department of Physics, Chemistry and Biology (IFM), Linköping University, Linköping, SE-58183, Sweden.

${ }^{3}$ Key Laboratory of Flexible Electronics (KLOFE) and Institute of Advanced Materials (IAM), Jiangsu National Synergetic Innovation Center for Advanced Materials (SICAM), Nanjing Tech University (Nanjing Tech), Nanjing, China

¥These authors contributed equally to this work.

Corresponding authors: sai.bai@liu.se;

tsong@suda.edu.cn;

jmcleod@suda.edu.cn;

bqsun@suda.edu.cn 


\begin{abstract}
Defect passivation has been demonstrated to be effective in improving the radiative recombination of charge carriers in perovskites, and consequently device performance of the resultant perovskite light-emitting diodes (LEDs). State-of-the-art useful passivation agents in perovskite LEDs are mostly organic chelating molecules, which, however, simultaneously sacrifice the charge transport properties and thermal stability of the resultant perovskite emissive layers, deteriorating the performance especially the operational stability of the devices. In this work, we demonstrate that lithium halides can efficiently passivate defects of halide vacancies and reduce trap state density, suppressing ion migration in perovskite films. Efficient green perovskite LEDs based on all inorganic $\mathrm{CsPbBr}_{3}$ perovskite with a peak external quantum efficiency of $16.2 \%$ as well as a high maximum brightness of $50270 \mathrm{~cd} \mathrm{~m}^{-2}$ are achieved. In addition, the device shows decent stability even under a brightness of $10^{4} \mathrm{~cd} \mathrm{~m}^{-2}$. We highlight the universal applicability of defect passivation using lithium halides, which enable us to improve the efficiency and stability of blue and red perovskite LEDs.
\end{abstract}

Keywords: passivation; non-radiative; lithium halide; surface defects; stability 


\section{Introduction}

Solution processed perovskite materials are emerging as celebrated semiconductors in light emitting diodes (LEDs) due to their excellent optoelectronic properties, such as widely tunable bandgap, high photoluminescence quantum efficiency (PLQE), and superior color purity. ${ }^{[1]}$ Since the first room-temperature perovskite LED reported by Friend and co-workers, rapid progress has been witnessed during the past few years. ${ }^{[2]}$ After great effort engineering device structure and perovskite film composition, the external quantum efficiencies (EQEs) of perovskite LEDs in the green, red, and nearinfrared regions have surpassed $20 \%{ }^{[1 b, 2 e, 3]}$

Despite this great success, further improvement in device efficiency and operational stability is necessary to accelerate commercialization of perovskite LEDs. It is well known that an operating LED generates significant Joule heating under a continuous electrical input, ${ }^{[4]}$ therefore, thermally stable perovskite films, e.g., all-inorganic perovskite $\mathrm{CsPbX}_{3}$ (where $\mathrm{X}=\mathrm{Cl}^{-}, \mathrm{Br}^{-}$or $\mathrm{I}^{-}$), are highly desirable for efficient and longterm operationally stable perovskite LEDs. ${ }^{[5]}$ Previous studies have shown that nonradiative recombination is one of the biggest problems in the perovskite emitters, as it deteriorates the efficiency and stability of LED devices. ${ }^{[6]}$ To overcome this issue, incorporating organic molecules consisting of electron-donating functional moieties have been widely applied to passivate the surface halide vacancies, enhancing the PLQEs of perovskite films. ${ }^{[2 b-d, 6 a]}$ Introducing large-size organic cations to form lowdimensional perovskite analogues with enhanced excitonic binding energy $\left(E_{b}\right)$ can also significantly enhance the radiative recombination rate in the resulting perovskite films. ${ }^{[2 \mathrm{~b}-\mathrm{d}]}$ However, these above mentioned strategies using organic molecules present drawbacks for perovskite LEDs with high efficiency as well as good stability, due to increased phase separation and thermal instability problems. ${ }^{[6 a, 6 b, 7]}$ In addition, the insulating nature of the introduced organic molecules also hinder charge transport in the perovskite films and is likely to generate more Joule heating during LED operation. ${ }^{[8]}$ Consequently, LEDs based on perovskite incorporated with organic molecules often show poor stability. It is clear that alternative passivation molecules 
are urgently needed to further improve the efficiency and stability of perovskite LEDs. In this work, we demonstrate that lithium halides ( $\mathrm{LiX}, \mathrm{X}=\mathrm{Cl}^{-}, \mathrm{Br}^{-}$or $\left.\mathrm{I}^{-}\right)$may be used as an alternative passivation agent, which enhances the radiative recombination in perovskite films without sacrificing stability. Within the halide-rich environment induced by adding rational $\mathrm{LiX}$ in the perovskite precursors, halide vacancies in the perovskite are well passivated without sacrificing conductivity and thermal stability of the resultant films. We present evidence that the $\mathrm{Li}^{+}$ions stay outside of the perovskite lattice and exhibit negligible influence on the energy level structure and crystallinity of the resulting perovskite films. Due to the greatly reduced defects, ion migration in the perovskite is also significantly suppressed. We select $\mathrm{LiBr}$ passivated $\mathrm{CsPbBr}_{3}$ as an example to synergistically investigate the effects of the inorganic passivation agent on the device performance of perovskite LEDs. The obtained green perovskite LEDs exhibit a high peak EQE of $16.2 \%$ and a high maximum brightness of $50270 \mathrm{~cd} \mathrm{~m}^{-2}$. In addition, the resulting LEDs exhibit an obviously improved operational lifetime even under a high brightness of $10^{4} \mathrm{~cd} \mathrm{~m}^{-2}$. We also demonstrate the general applicability of the inorganic passivation strategy to different perovskite systems, enabling the fabrication of mixed-halide blue and red perovskite LEDs with improved efficiency and stability.

\section{Results and Discussion}

\section{Passivation Effects of Lithium Bromide}

Different perovskite precursors were prepared by varying the molar ratios of $\mathrm{LiBr}$ and $\mathrm{CsPbBr}_{3}$ (see detailed recipes in Table S1 in Supporting Information ST4, 20\% LiBr is the maximum concentration). $\mathrm{Cs} \mathrm{PbBr}_{3}$ with $0 \% \mathrm{LiBr}$ and trace amount of crown is defined as reference sample. We do not observe any shift in the photoluminescence (PL) and absorption spectra of the LiBr-containing perovskite films, as shown in Figure S1, suggesting that $\mathrm{LiBr}$ does not influence the band gap of perovskite films. This is also confirmed by ultraviolet photoelectron spectroscopy (UPS) measurement where there is neglectable offset of work function (WF) and valence band maximum (VBM) 
between reference perovskite and 20\% LiBr one, as shown in Figure S2. However, we do measure dramatic improvement in the PL intensity of the LiBr-containing perovskite films in comparison to the reference sample (Figure S1a), which indicates greatly reduced trap states in the films with the addition of $\mathrm{LiBr}$.

To quantitatively analyze the trap density in the perovskite films, we fabricate a simple hole-only device with an architecture of ITO, poly (vinylcarbazole) (PVK), perovskite, and molybdenum oxide/silver $\left(\mathrm{MoO}_{\mathrm{x}} / \mathrm{Ag}\right)$ (Figure S3a). We extract the trap density through a widely reported space-charge limited current (SCLC) model (see more discussions in Supporting Information ST1). ${ }^{[9]}$ According to the $J$ - $V$ curves in Figure 1a, the calculated trap density $n_{t}$ are $1.4 \times 10^{17} \mathrm{~cm}^{-3}$ (reference), $1.0 \times 10^{17} \mathrm{~cm}^{-3}(10 \% \mathrm{LiBr})$ and $6.6 \times 10^{16} \mathrm{~cm}^{-3}(20 \% \mathrm{LiBr})$, respectively. Consequently, a high PLQE of $\sim 30 \%$ for the $20 \% \mathrm{LiBr}$-incorporated perovskite film is achieved. Moreover, no trap-filling behavior can be observed in $20 \% \mathrm{LiBr}$-incorporated film while the excitation intensity is increased from 0.1 to $200 \mathrm{~mW} \mathrm{~cm}{ }^{-2}$, however a linear increase in PLQE is observed in the reference and $10 \% \mathrm{LiBr}$-incorporated films under the same conditions. These results suggest suppressed trap-mediated non-radiative recombination in the perovskite film with $20 \% \mathrm{LiBr}$, which is also verified by the much increased PL lifetime in the time-correlated single photon counting (TCSPC) measurements (Figure 1c). ${ }^{[3 a]}$

Figure 1d shows the X-ray diffraction (XRD) patterns of the perovskite films. We observe stronger diffraction peaks for (100), (110), and (200) crystal planes in perovskite film consisting of higher concentration of $\mathrm{LiBr}$, suggesting an improved crystallinity with $\mathrm{LiBr}$ incorporation, consistent with previous studies using alkaline metal salts in perovskite materials. ${ }^{[10]} \mathrm{We}$ anticipate that $\mathrm{LiBr}$ is likely to be confined at the perovskite surface rather than occupying the perovskite lattice, since no parasitic diffraction peaks and lattice strain can be observed from the XRD results. The hypothesis is further confirmed by the negligible difference among the fitted results on the lattice strain for different perovskite films (Figure S4) using a Willamson-Hall plot extracted from the XRD data. ${ }^{[1]}$ There is no peak which is ascribed to $\mathrm{LiBr}$ due to its limited amount ratio in the perovskite film. In addition, no XRD peak can be detected from a LiBr film deposited from its solution on Si substrate, as shown in Figure S5. X- 
ray photoelectron spectroscopy (XPS) measurement also shows that there is no peak shift for $\mathrm{Cs} 3 \mathrm{~d}, \mathrm{~Pb} 4 \mathrm{f}$ and $\mathrm{Br} 3 \mathrm{~d}$ of perovskite films with and without $\mathrm{LiBr}$ incorporation, which reveals that $\mathrm{Li}$ has no interactions with $\mathrm{Cs}, \mathrm{Pb}$ or $\mathrm{Br}$, as shown in Figure $\mathbf{S 6}$. We further prove that incorporating $\mathrm{LiBr}$ does not form a low-dimensional perovskite, evidenced by the synchrotron-based grazing incidence X-ray diffraction (GIXRD) measurements (Figure S7), showing no additional peaks at low $q$ values. In contrast to the observed obvious pinholes in the reference samples, the LiBr-incorporated perovskite films show compact and pin-hole free film morphology, especially for the $20 \% \mathrm{LiBr}$-incorporated one, as shown by the scanning electron microscopy (SEM) images presented in Figure S8.

\section{Passivation Mechanism of Lithium Bromide}

We carried out temperature-dependent PL measurement and extract exciton binding energies $\left(E_{b}\right)$ of $19.9 \mathrm{meV}$ and $30.1 \mathrm{meV}$ for the reference and $20 \% \mathrm{LiBr}$-contained perovskite films, respectively (Figure S3b-d). Consistent with previous studies, ${ }^{[2 c, 12]}$ we anticipate that the improved PL in LiBr-incorporated perovskite films should be from the passivation effects rather than the $E_{b}$ difference since the latter is relatively low compared to the strong PL enhancement observed. To obtain in-depth understanding of the passivation mechanism of $\mathrm{LiBr}$, we perform density functional theory (DFT) calculations on $\mathrm{CsPbr}_{3}$ slabs (5-unit cells thick, see Supporting Information ST2 for details). We examined (100) surfaces, terminated by flat $\mathrm{PbBr}_{2}$ layers ("flat"), dangling $\mathrm{Br}$ (“Br"), a $\mathrm{CsBr}$ layer (“CsBr"), a $\mathrm{LiBr}$ layer (“'LiBr"), hanging Cs ("Cs"), and hanging Li ("Li"). The optimized geometries of these structures are shown in Figure 3a. The basic perovskite structure is generally unaffected by the choice of surface termination, except in the case of $\mathrm{LiBr}$ and $\mathrm{Li}$ (here the $\mathrm{LiPbBr}_{3}$ and $\mathrm{LiPBr}_{2}$ surface layers, respectively). Li has been pulled away from the $\mathrm{CsPbBr}_{3}$ bulk, to the extent that Li-terminated surfaces actually resemble an almost flat $\mathrm{LiPbBr}_{2}$ layer physiosorbed on a $\mathrm{CsBr}$-terminated $\mathrm{CsPbBr}_{3}$ perovskite surface, as shown in the Figure 2a. The calculation results suggest that $\mathrm{Li}$ atoms possess little chance to enter the perovskite lattice.

The surface energy (per single perovskite cell at the surface) relative to bulk is relatively 
small for flat and Br-terminated surfaces, and is actually negative for CsBr-terminated surfaces, as shown in Figure 2b. However, the surface energy for LiBr-, Cs-, and Literminated surfaces is significantly larger, suggesting that these structures are unlikely to form and that separate phases are more likely. The negative energy for the $\mathrm{CsBr}$ terminated surface (this energy is relative to separate bulk phases of $\mathrm{CsPbBr}_{3}$ and $\mathrm{CsBr}$ ) suggest that this is the most thermodynamically favorable surface.

The electronic density of states (DOS) of all structures possesses a shape that is dominated by the bulk perovskite, as shown in Figure 2c, with a narrow valence band of primarily Br-4p states and a gradual conduction band onset with a calculated band gap between $1.7 \mathrm{eV}$ and $1.8 \mathrm{eV}$ (this is an underestimate of the true band gap). However, the choice of surface termination does have a significant influence on both the presence of mid-gap states and the work function. A Br-terminated surface provides acceptor states just above the valence band maximum (VBM), while a Cs- or Li-terminated surface provides donor states just below the conduction band minimum (CBM). These calculations suggest that the thermodynamically-favored CsBr-terminated surface is also the best state for optoelectronics, providing negligible modification to the electronic structure of bulk $\mathrm{CsPbBr}_{3}$ and, most importantly, providing no mid-gap states that may assist non-radiative recombination. A LiBr-terminated surface similarly provides the same electronic structure, but the high surface energy suggests that this structure is unlikely to form.

As a result, the key function of $\mathrm{LiBr}$ is simply providing a Br-rich environment to prevent free $\mathrm{Cs}^{+}$ions from aggregating at the surface and to passivate $\mathrm{Br}^{-}$vacancies to reduce non-radiative recombination centers. A significant amount $\mathrm{Li}^{+}$may not be present in the resultant films, even at the surface. Attempting to provide a Br-rich environment by increasing the concentration of $\mathrm{CsBr}$ in the precursors instead favors the formation of a parasitic and non-luminescent $\mathrm{Cs}_{4} \mathrm{PbBr}_{6}$ phase, ${ }^{[8 b]}$ which is also proved by absorption spectra as shown in Figure S9. As there is no physically possible $\mathrm{Li}_{\mathrm{x}} \mathrm{PbBr}_{\mathrm{y}}$ phases to compete with perovskite formation, adding $\mathrm{LiBr}$ to the precursors provides a $\mathrm{Br}$-rich environment without this risk. We also investigated $\mathrm{CsPbBr}_{3}$ slabs terminating with other alkaline metal bromide, as shown in Figure S10a. The surface 
energies for $5 \mathrm{CsPbBr}_{3}$ layered $\mathrm{LiBr}$ - and $\mathrm{CsBr}$-terminated slabs are repeated in Figure S10b, along with those of $\mathrm{NaBr}-, \mathrm{KBr}-$, and $\mathrm{RbBr}$-terminated slabs. It is clear the major influence of the different halide salts is to reduce the surface energy. This result again indicates that $\mathrm{Li}$ is most unlikely to occupy the A-site in perovskite. Other metal ions $(\mathrm{Na}, \mathrm{K}, \mathrm{Rb})$ have greater chance to enter the lattice due to their larger size, which may deteriorate perovskite if not well controlled. ${ }^{[10 b, 13]}$ We prepared $\mathrm{CsPbBr}_{3}$ films with excess $\mathrm{NaBr}, \mathrm{KBr}, \mathrm{RbBr}$ and $\mathrm{CsBr}$. Only $\mathrm{LiBr}$-based one showed a strong PL emission, as shown in Figure S10c, which confirmed our assumption.

According to the above discussion, it is clear that $\mathrm{Li}^{+}$does not enter the $\mathrm{Cs} \mathrm{PbBr}_{3}$ lattice. Here, we propose a model for the passivation of $\mathrm{LiBr}$, as illustrated in Figure 3a. The incorporation $\mathrm{LiBr}$ can easily bring in excess $\mathrm{Br}^{-}$ions. In this case, most of surface defects from $\mathrm{Br}^{-}$vacancies are filled by the $\mathrm{Br}^{-}$ions, removing these trap states and leading to great enhancement in radiative recombination, which is also the mechanism for phenethylamine hydrobromide $(\mathrm{PEABr})$ as previously reported. ${ }^{[6]}$ Due to the negligible concentration of organic compounds, outstanding thermal stability is achieved for LiBr-incorporated perovskite films. For 20\% LiBr-incorporated sample, the PL intensity remains essentially constant even after continuously annealing at $120{ }^{\circ} \mathrm{C}$ for 24 h, as shown in Figures 3b, c and also Figure S11. As a point of comparison, we also fabricate an efficient PEABr based quasi-2D perovskite film using a widely reported method. ${ }^{[6 a]}$ The PEABr based perovskite film exhibits poor thermal tolerance under the same measurement conditions, with the PL almost disappearing after annealing for only $40 \mathrm{~min}$ at same temperature. These thermal stability measurements indicate an obvious advantage of using inorganic $\mathrm{LiBr}$ to passivate $\mathrm{Cs} \mathrm{PbBr}_{3}$ to achieve perovskite LEDs with high efficiency and enhanced operational stability.

\section{Devices efficiency and operational Stability of perovskite LEDs}

We fabricate perovskite LEDs with a device architecture of indium tin oxide (ITO), nickel oxide $\left(\mathrm{NiO}_{\mathrm{x}}\right)$, poly (9, 9-dioctyl-fluorene-co-N-(4-butylphenyl) dipheny-lamine) (TFB), PVK, perovskite, 1,3,5-tris(1-phenyl-1H-benzi-midazol-2-yl)-benzene (TPBi), and $\mathrm{LiF}$ and $\mathrm{Al}$, as shown in the cross-sectional SEM image in Figure 4a. The energy band structures of each layer are shown in Figure S12, these energy values are extracted 
from literatures. ${ }^{[2 b, 2 d, 14]} 18$-crown-6 was added in the solution to improve the morphology of the perovskite film and minimize the leakage current. Perovskite films without crown were also fabricated. ${ }^{[15]}$ As shown in Figure S13a, the film with LiBr added also shows enhanced PL which further proves the passivation effect of $\mathrm{LiBr}$. However, as shown in Figure S13c, d, the film without crown exhibits a significant amount of pin-holes. As a result, the devices without crown in Figure S13b show high current density but low luminance. All the devices with crown added display same electroluminescence (EL) peaks at $520 \mathrm{~nm}$ (Figure 4b). The LiBr-incorporated devices exhibit greatly enhanced brightness under the same driving voltage, which agrees well with the previously discussed enhancement of the PLQE in the LiBr-incorporated perovskite films. As a result, 20\% LiBr-incorporated device shows a decreased turn-on voltage of $2.4 \mathrm{~V}\left(V_{T}\right.$, defined as the voltage required to drive the device with $L=1 \mathrm{~cd} \mathrm{~m}^{-}$ ${ }^{2}$, see Figure 4c). The maximum brightness for perovskite LEDs is $26230 \mathrm{~cd} \mathrm{~m}^{-2}$ (reference), $35400 \mathrm{~cd} \mathrm{~m}^{-2}(10 \% \mathrm{LiBr})$, and $50278 \mathrm{~cd} \mathrm{~m}^{-2}$ (20\% LiBr), respectively. The peak EQEs are $2.83 \%, 7.92 \%$ and $16.2 \%$ for the reference, the $10 \% \mathrm{LiBr}$ and $20 \% \mathrm{LiBr}-$ incorporated devices, respectively (Figure 4d). Figure 4e shows an EQE histogram from 25 individual 20\% LiBr-incorporated devices, gives an average EQE value of 14.9\%. Table 2 summarizes the detail electrical output of reference and LiBrincorporated devices. Note that trap-filling exists an LED device is driven under low current density, as most of the injected charge carriers will fill the defects first. ${ }^{[16]}$ Significant improvement of EQE under higher current density is observed in $20 \% \mathrm{LiBr}$ incorporated device; while reference and $10 \% \mathrm{LiBr}$ based devices with more defects show gradually increasing EQE during device operation, consistent with a longer time is needed to fill up the larger amounts of defects.

Due to the superior thermal stability of LiBr-incorporated perovskite films, the obtained LEDs exhibit improved operational stability. For comparison, we also fabricate a quasi2D LED with widely used organic molecule of PEABr. As shown in Figure S14a-d, the PEABr-based LED shows significant degradation after the first test, while the LiBrincorporated perovskite LED retains a high efficiency output. We used an infrared camera to record the surface temperature of the devices during testing, as shown in 
Figure S14c and d. At the highest luminance, the LiBr-incorporated perovskite LED exhibits a higher temperature than that of the PEABr-based one. However, the LiBrincorporated perovskite LED still displays a high efficiency even at these elevated temperatures, which correlates with the improved thermal tolerance.

As a benefit from the much-reduced non-radiative recombination and improved thermal tolerance in the LiBr-incorporated perovskite films, the LiBr-incorporated perovskite LEDs also show suppressed ion migration and longer operational lifetime. As shown in Figure S15, the forward and backward $J-V$ scan of $20 \% \mathrm{LiBr}$ device shows less hysteresis, which should be correlated with suppressed ion migration behavior. We measure the operational stability of the LED devices in ambient (humidity 80\%) conditions with simple epoxy encapsulation. We set the initial brightness $\left(L_{0}\right)$ of $10^{4} \mathrm{~cd}$ $\mathrm{m}^{-2}$ and record the luminance decay over time (Figure 4f). The 20\% LiBr-incorporated device has a prolonged lifetime (defined as $T_{50}$, luminance decay to half of its initial value) of $\sim 6.5 \mathrm{~min}$ compared to the reference device one of only $\sim 1.5 \mathrm{~min}$. There is no EL peak shift when the $20 \%$ - $\mathrm{LiBr}$ device operates under continuous current at a $L_{0}$ of $10^{4} \mathrm{~cd} \mathrm{~m}^{-2}$ as shown in Figure S16.

Furthermore, we predicted the $T_{50}$ at $100 \mathrm{~cd} \mathrm{~m}^{-2}$ and $1000 \mathrm{~cd} \mathrm{~m}^{-2}$ of this device to be over $108 \mathrm{~h}$ and $3.4 \mathrm{~h}$ respectively, by using the relation $L_{0}{ }^{n} T_{50}=$ constant (here assuming the acceleration factor of $\left.n=1.5^{[3 \mathrm{~b}]}\right)$, which is comparable to recently reported record green perovskite LEDs. ${ }^{[3 \mathrm{~b}, 17]}$. However, when we set an initial brightness at $100 \mathrm{~cd} \mathrm{~m}^{-}$ 2 and $1000 \mathrm{~cd} \mathrm{~m}^{-2}$, our best LiBr-incorporated device only exhibits a lifetime of $\sim 21 \mathrm{~h}$ and $\sim 1.2$ h, respectively (Figure S17). This may be because simple epoxy encapsulation method is not good enough to fully isolate the perovskite LED from water, so that the LED may degrade when operating in air for a long time. As expected, PEABr-based perovskite LED has shown much poorer stability as shown in Figure S18 demonstrating around $1.2 \mathrm{~h}$ half-lifetime at $\sim 100 \mathrm{~cd} \mathrm{~m}^{-2}$.

\section{General passivation of Multi-colored Perovskite LEDs with Lithium Halide}

We highlight that the LiX incorporation strategy is a general strategy for multi-color perovskite films as well as LED devices. To demonstrate this, blue $\left(\mathrm{CsPbBCl}_{2}\right)$ and red $\left(\mathrm{CsPBrI}_{2}\right)$ perovskite films (see recipe in Table S1-3) were also fabricated. These 
reference films show extremely low PL emission under a $365 \mathrm{~nm}$ UV lamp, as shown in Figure S19. Very similar to the $\mathrm{LiBr}$-incorporated green $\mathrm{CsPbBr}_{3}$ perovskite films (labeled as "G" hereafter), LiCl-incorporated blue $\mathrm{CsPbBrCl}_{2}$ (labeled as "B1"), $\mathrm{LiBr}$ incorporated blue $\mathrm{CsPbBrCl}_{2}$ (labeled as "B2") and $\mathrm{LiI}$-incorporated red $\mathrm{CsPbBrI}_{2}$ (labeled as "R") films show dramatically enhanced PL emission (Figure 5b inset) and PLQE value (Figure 5b), which can significantly reduce the non-radiative recombination. We can use LiX passivation strategy to enhance the PL emission of halide-mixed perovskite films from $480 \mathrm{~nm}$ to $680 \mathrm{~nm}$ (Figure 5a), paving the way for practical applications in future.

Perovskite LEDs were also fabricated with the same device architecture to demonstrate the effect of LiX passivation on the performance of multi-color devices. Figure 5c and S20 show EL peaks and pictures of working different color LED devices (all with device area of $1 \times 1 \mathrm{~cm}^{2}$ ). All LEDs device show uniform light emission following a Lambertian profile (Figure S21). The Commission Internationale de L'Eclairage (CIE) coordination of B1, G and R perovskite LEDs are plotted in Figure 5d, which covers a large area in the CIE chromaticity diagram. This demonstrates the huge potential of these devices to be integrated into high quality display applications. The efficiency of LiCl-incorporated B1 and LiI-incorporated red devices also show same trend as the LiBr-incorporated green one. An improvement in both brightness and efficiency can be observed with LiX (Figure S22, 23). The electrical output of different perovskite LEDs are summarized in Table S4. The peak EQEs for LEDs based on LiCl-incorporated blue and LiI-incorporated perovskite are $0.96 \%$ (B1), 2.1\% (B2) and 2.0\% (R), respectively, as shown in Figure S23.

\section{Conclusion}

In summary, we have demonstrated a novel defect passivation strategy using inorganic lithium halides for perovskite emissive layers. This passivation strategy not only reduces non-radiative recombination, but also retains the thermal stability of inorganic perovskite films. An efficient green perovskite LED yields a peak luminescence of $50278 \mathrm{~cd} \mathrm{~m}^{-2}$ and a peak EQE of $16.2 \%$. Moreover, due to the much-reduced defects in the perovskite films, the obtained LEDs exhibit a much longer operational lifetime even 
under a high luminance of $10^{4} \mathrm{~cd} \mathrm{~m}^{-2}$. The general applicability of the defect passivation strategy enables us to achieve efficient and spectrally stable multi-colored LEDs with mixed-halide perovskite films. Our findings provide a promising avenue to reduce nonradiative recombination to further improve the efficiency and stability in perovskitebased optoelectronics.

\section{Acknowledgements}

This work was supported by the National Key Research and Development Program of China (2016YFA0202402), the National Natural Science Foundation of China (61974098, 61974098, 61674108), Jiangsu High Educational Natural Science Foundation (18KJA430012), the Priority Academic Program Development of Jiangsu Higher Education Institutions, the 111 program and Collaborative Innovation Center of Suzhou Nano Science and Technology (NANO-CIC). Z.Y.T. also thanks the financial support from China Scholarship Council (No. 201806920071) and Postgraduate Research and Practice Innovation Program of Jiangsu Province (KYCX18_2504).

\section{Conflict of Interest}

The authors declare no conflict of interest.

\section{Reference}

[1] a) Q. Chen, J. Wu, X. Ou, B. Huang, J. Almutlaq, A. A. Zhumekenov, X. Guan, S. Han, L. Liang, Z. Yi, J. Li, X. Xie, Y. Wang, Y. Li, D. Fan, D. B. L. Teh, A. H. All, O. F. Mohammed, O. M. Bakr, T. Wu, M. Bettinelli, H. Yang, W. Huang, X. Liu, Nature 2018, 561, 88-93; b) T. Chiba, Y. Hayashi, H. Ebe, K. Hoshi, J. Sato, S. Sato, Y.-J. Pu, S. Ohisa, J. Kido, Nat. Photonics 2018, 12, 681-687; c) X. Zhang, H. Lin, H. Huang, C. Reckmeier, Y. Zhang, W. C. H. Choy, A. L. Rogach, Nano Lett. 2016, 16, 1415-1420; d) Q. Zhou, Z. Bai, W.-g. Lu, Y. Wang, 
B. Zou, H. Zhong, Adv. Mater. 2016, 28, 9163-9168; e) Q. A. Akkerman, V. D’Innocenzo, S. Accornero, A. Scarpellini, A. Petrozza, M. Prato, L. Manna, J. Am. Chem. Soc. 2015, 137, 10276-10281.

[2] a) Z.-K. Tan, R. S. Moghaddam, M. L. Lai, P. Docampo, R. Higler, F. Deschler, M. Price, A. Sadhanala, L. M. Pazos, D. Credgington, F. Hanusch, T. Bein, H. J. Snaith, R. H. Friend, Nat. Nanotechnol. 2014, 9, 687; b) N. Wang, L. Cheng, R. Ge, S. Zhang, Y. Miao, W. Zou, C. Yi, Y. Sun, Y. Cao, R. Yang, Y. Wei, Q. Guo, Y. Ke, M. Yu, Y. Jin, Y. Liu, Q. Ding, D. Di, L. Yang, G. Xing, H. Tian, C. Jin, F. Gao, R. H. Friend, J. Wang, W. Huang, Nat. Photonics 2016, 10, 699; c) M. Yuan, L. N. Quan, R. Comin, G. Walters, R. Sabatini, O. Voznyy, S. Hoogland, Y. Zhao, E. M. Beauregard, P. Kanjanaboos, Z. Lu, D. H. Kim, E. H. Sargent, Nat. Nanotechnol. 2016, 11, 872; d) Z. Xiao, R. A. Kerner, L. Zhao, N. L. Tran, K. M. Lee, T.-W. Koh, G. D. Scholes, B. P. Rand, Nat. Photonics 2017, 11, 108; e) W. Xu, Q. Hu, S. Bai, C. Bao, Y. Miao, Z. Yuan, T. Borzda, A. J. Barker, E. Tyukalova, Z. Hu, M. Kawecki, H. Wang, Z. Yan, X. Liu, X. Shi, K. Uvdal, M. Fahlman, W. Zhang, M. Duchamp, J.-M. Liu, A. Petrozza, J. Wang, L.-M. Liu, W. Huang, F. Gao, Nat. Photonics 2019, 13, 418-424.

[3] a) Y. Cao, N. Wang, H. Tian, J. Guo, Y. Wei, H. Chen, Y. Miao, W. Zou, K. Pan, Y. He, H. Cao, Y. Ke, M. Xu, Y. Wang, M. Yang, K. Du, Z. Fu, D. Kong, D. Dai, Y. Jin, G. Li, H. Li, Q. Peng, J. Wang, W. Huang, Nature 2018, 562, 249-253; b) K. Lin, J. Xing, L. N. Quan, F. P. G. de Arquer, X. Gong, J. Lu, L. Xie, W. Zhao, D. Zhang, C. Yan, W. Li, X. Liu, Y. Lu, J. Kirman, E. H. Sargent, Q. 
Xiong, Z. Wei, Nature 2018, 562, 245-248.

[4] a) Z. Wei, A. Perumal, R. Su, S. Sushant, J. Xing, Q. Zhang, S. T. Tan, H. V. Demir, Q. Xiong, Nanoscale 2016, 8, 18021-18026; b) J. C. Yu, D. W. Kim, D. B. Kim, E. D. Jung, J. H. Park, A.-Y. Lee, B. R. Lee, D. Di Nuzzo, R. H. Friend, M. H. Song, Adv. Mater. 2016, 28, 6906-6913.

[5] H. Wang, X. Zhang, Q. Wu, F. Cao, D. Yang, Y. Shang, Z. Ning, W. Zhang, W. Zheng, Y. Yan, S. V. Kershaw, L. Zhang, A. L. Rogach, X. Yang, Nat. Commun. 2019, 10,665 .

[6] a) M. Ban, Y. Zou, J. P. H. Rivett, Y. Yang, T. H. Thomas, Y. Tan, T. Song, X. Gao, D. Credgington, F. Deschler, H. Sirringhaus, B. Sun, Nat. Commun. 2018, 9, 3892; b) J. Song, T. Fang, J. Li, L. Xu, F. Zhang, B. Han, Q. Shan, H. Zeng, Adv. Mater. 2018, 30, 1805409; c) Z. Yuan, Y. Miao, Z. Hu, W. Xu, C. Kuang, K. Pan, P. Liu, J. Lai, B. Sun, J. Wang, S. Bai, F. Gao, Nat. Commun. 2019, 10, 2818

[7] H. Cho, Y.-H. Kim, C. Wolf, H.-D. Lee, T.-W. Lee, Adv. Mater. 2018, 30, 1704587.

[8] a) F. Zhang, D. H. Kim, H. Lu, J.-S. Park, B. W. Larson, J. Hu, L. Gao, C. Xiao, O. G. Reid, X. Chen, Q. Zhao, P. F. Ndione, J. J. Berry, W. You, A. Walsh, M. C. Beard, K. Zhu, J. Am. Chem. Soc. 2019, 141, 5972-5979; b) L. Wu, H. Hu, Y. Xu, S. Jiang, M. Chen, Q. Zhong, D. Yang, Q. Liu, Y. Zhao, B. Sun, Q. Zhang, Y. Yin, Nano Lett. 2017, 17, 5799-5804.

[9] Z. Liu, J. Hu, H. Jiao, L. Li, G. Zheng, Y. Chen, Y. Huang, Q. Zhang, C. Shen, 
Q. Chen, H. Zhou, Adv. Mater. 2017, 29, 1606774.

[10] a) O. A. Syzgantseva, M. Saliba, M. Grätzel, U. Rothlisberger, J. Phys. Chem. Lett. 2017, 8, 1191-1196; b) J. K. Nam, S. U. Chai, W. Cha, Y. J. Choi, W. Kim, M. S. Jung, J. Kwon, D. Kim, J. H. Park, Nano Lett. 2017, 17, 2028-2033.

[11] E. T. Hoke, D. J. Slotcavage, E. R. Dohner, A. R. Bowring, H. I. Karunadasa, M. D. McGehee, Chem. Sci. 2015, 6, 613-617.

[12] C. Wu, Y. Zou, T. Wu, M. Ban, V. Pecunia, Y. Han, Q. Liu, T. Song, S. Duhm, B. Sun, Adv. Funct. Mater. 2017, 27, 1700338.

[13] M. Saliba, T. Matsui, K. Domanski, J.-Y. Seo, A. Ummadisingu, S. M. Zakeeruddin, J.-P. Correa-Baena, W. R. Tress, A. Abate, A. Hagfeldt, M. Grätzel, Science 2016, 354, 206-209.

[14] J. You, L. Meng, T.-B. Song, T.-F. Guo, Y. Yang, W.-H. Chang, Z. Hong, H. Chen, H. Zhou, Q. Chen, Y. Liu, N. De Marco, Y. Yang, Nat. Nanotechnol. 2015, 11,75 .

[15] C. Wu, T. Wu, Y. Yang, J. A. McLeod, Y. Wang, Y. Zou, T. Zhai, J. Li, M. Ban, T. Song, X. Gao, S. Duhm, H. Sirringhaus, B. Sun, ACS Nano 2019, 13, 1645 1654.

[16] a) Z. Yuan, Z. Wu, S. Bai, Z. Xia, W. Xu, T. Song, H. Wu, L. Xu, J. Si, Y. Jin, B. Sun, Adv. Energy Mater. 2015, 5, 1500038; b) H. T. Nicolai, M. M. Mandoc, P. W. M. Blom, Phys. Rev. B 2011, 83, 195204.

[17] J. Wang, C. Song, Z. He, C. Mai, G. Xie, L. Mu, Y. Cun, J. Li, J. Wang, J. Peng, Y. Cao, Adv. Mater. 2018, 30, 1804137. 

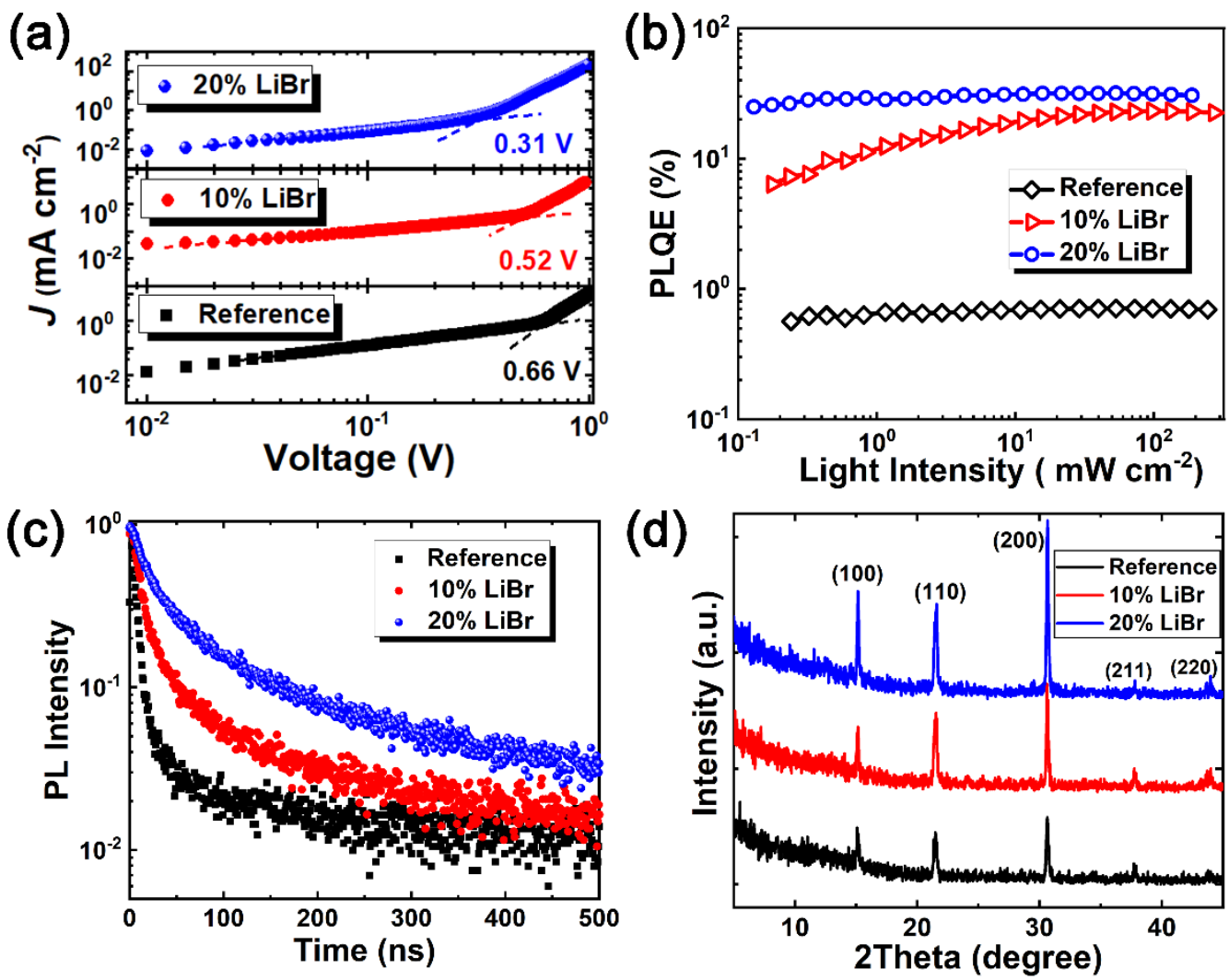

Figure 1 Reduced non-radiative recombination with $\mathrm{LiBr}$ passivation. (a) $\mathrm{J}-\mathrm{V}$ curves of hole-only devices with reference and LiBr-incorporated perovskite films; (b) Excitation-intensity-dependent $\mathrm{PLQE}$ of $\mathrm{CsPbBr}_{3}$ perovskite films with different concentrations of $\mathrm{LiBr}$; (c) TCSPC probed PL lifetime of reference and $\mathrm{LiBr}$ incorporated perovskite films under the excitation of $370 \mathrm{~nm}$ laser pumping; (d) XRD spectra of perovskite with different $\mathrm{LiBr}$ molar ratios $(0 \%, 10 \%, 20 \%)$. 
(a)
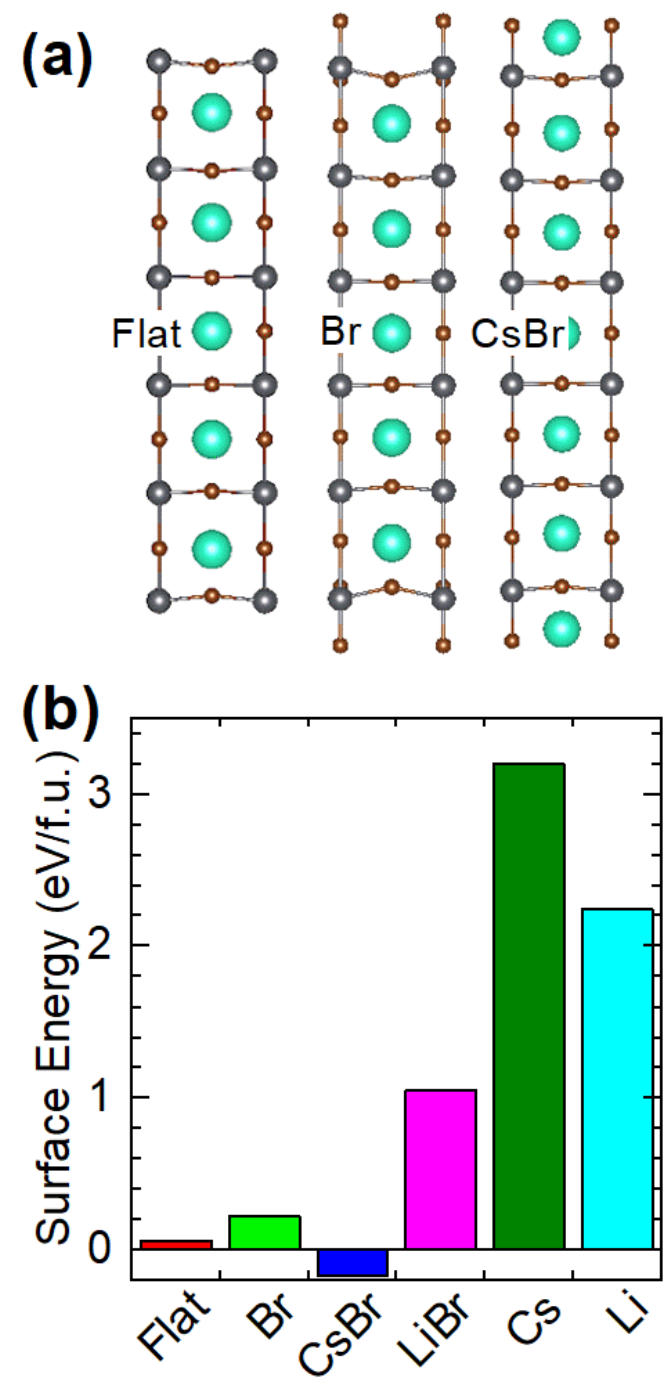
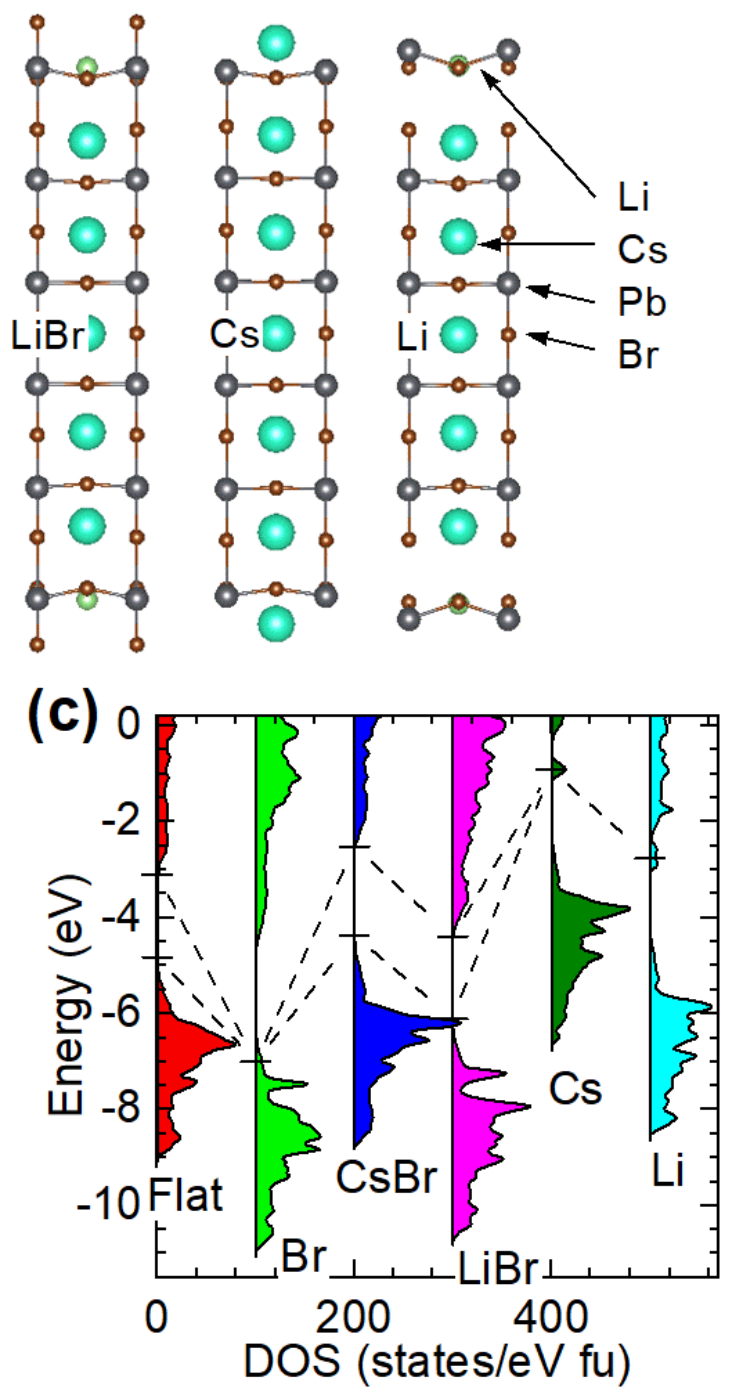

Figure 2 DFT simulation for $\mathrm{LiBr}$ passivation mechanism. (a) Optimized flat, Brterminated, CsBr-terminated, LiBr-terminated, Cs-terminated and Li-terminated perovskite structures, (b) Calculated surface energy for $\mathrm{CsPbBr}_{3}$ slabs surface energy (per single perovskite cell at the surface) relative to bulk with different surface terminations, (c) DOS of 5-layered $\mathrm{CsPbBr}_{3}$ slabs with different surface terminations. In (c) the VBM and CBM are indicated by the horizontal lines, connected by dotted lines as a guide for the eye. 


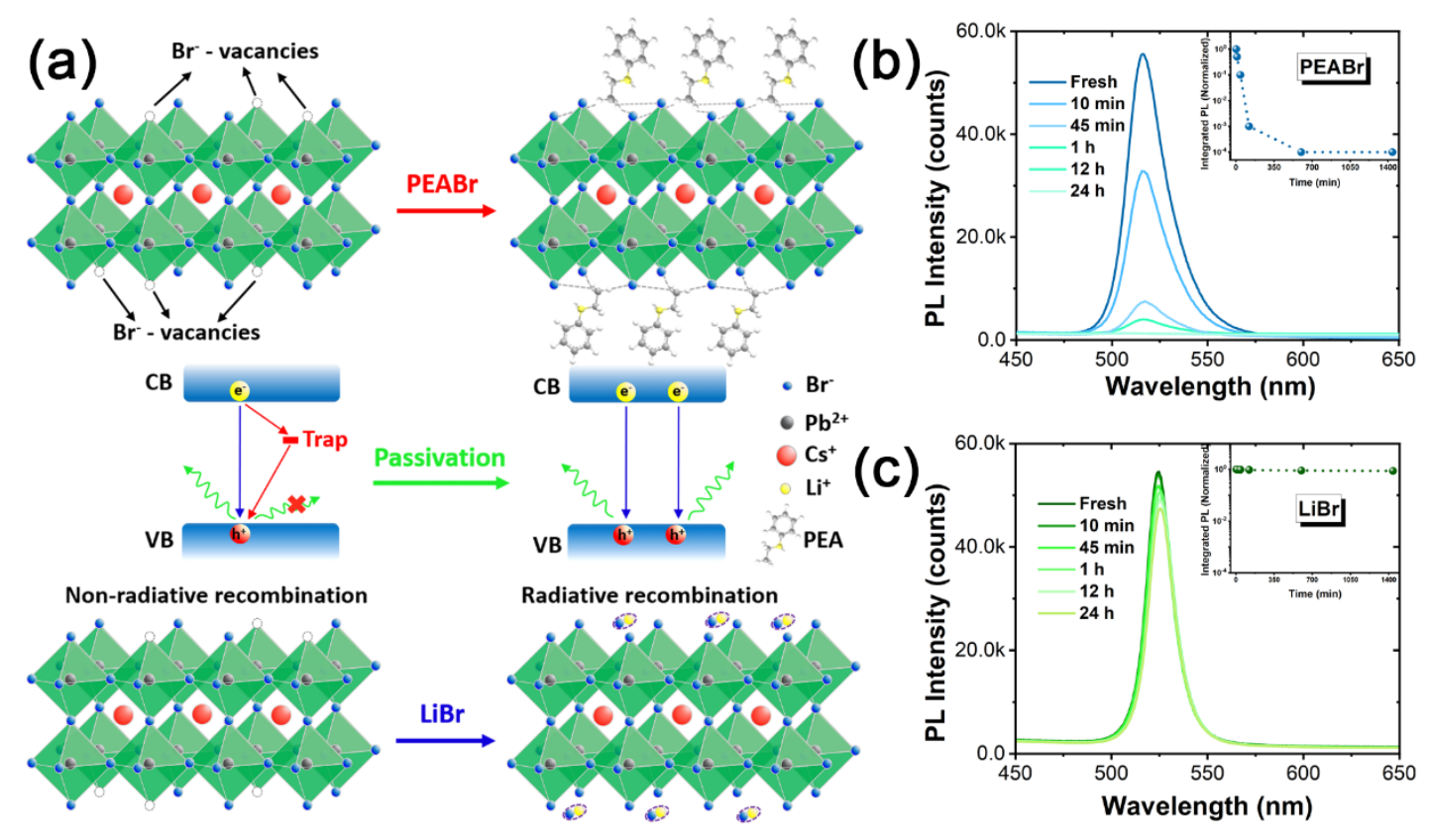

Figure 3 Stability of perovskite films with organic and inorganic passivation agent.

(a) Schematics of surface halide vacancies of $\mathrm{CsPbrr}_{3}$ perovskite films and passivated by organic molecule of PEABr and inorganic molecule of LiBr. Time-dependent PL spectra and integrated PL intensities (inset) of perovskite films annealed at $120^{\circ} \mathrm{C}$, (b) 40\% PEABr incorporated and (c) 20\% LiBr-incorporated perovskite films. 
(a)

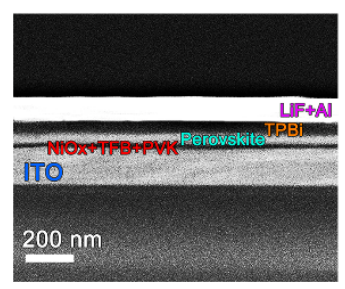

(d)

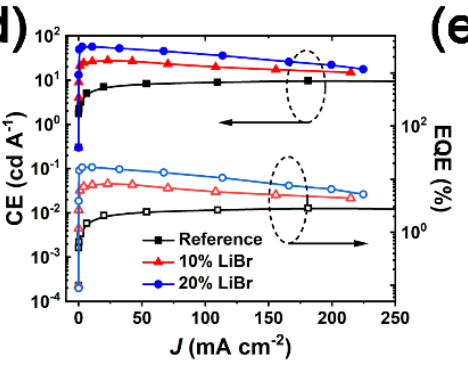

(b)

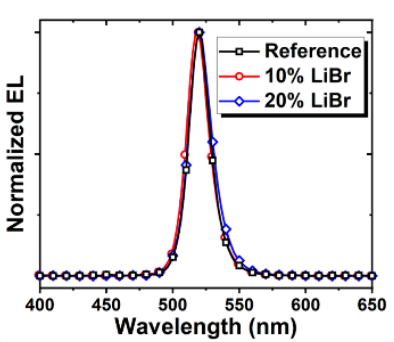

(e)

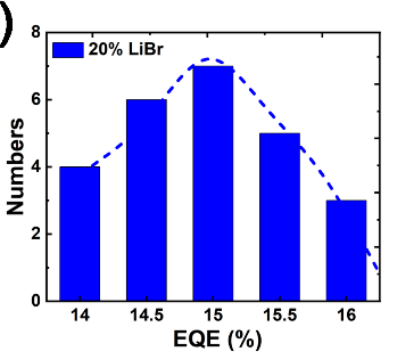

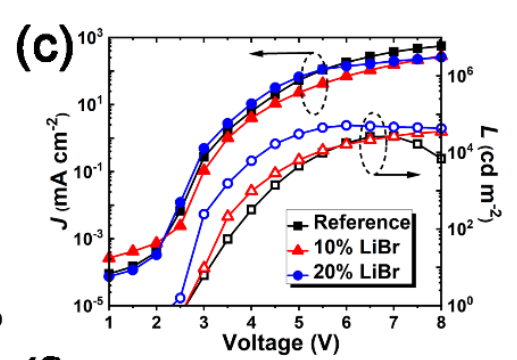

(f)

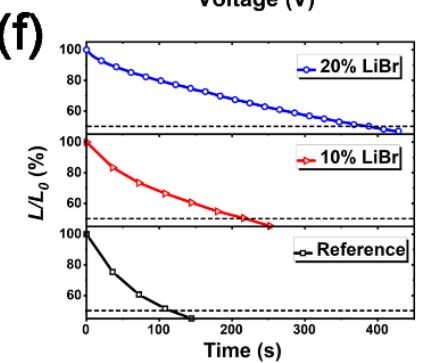

Figure 4 LED devices performance. (a) Cross-sectional SEM image of a 20\% $\mathrm{LiBr}$ perovskite based perovskite LED; (b) EL peaks of reference, 10\%, and 20\% LiBrincorporated perovskite LEDs; (c) $J-V-L$ curves of reference, $10 \%$, and $20 \% \mathrm{LiBr}$ incorporated perovskite LEDs; (d) CE and EQE against current density of reference, 10\%, and 20\% LiBr-incorporated perovskite LEDs; (e) EQE distribution of 25 individual devices for 20\% LiBr-incorporated based perovskite LEDs; (f) Operational stability of LEDs under constant current measured with an initial luminance of $\sim 10^{4} \mathrm{~cd}$ $\mathrm{m}^{-2}$. 

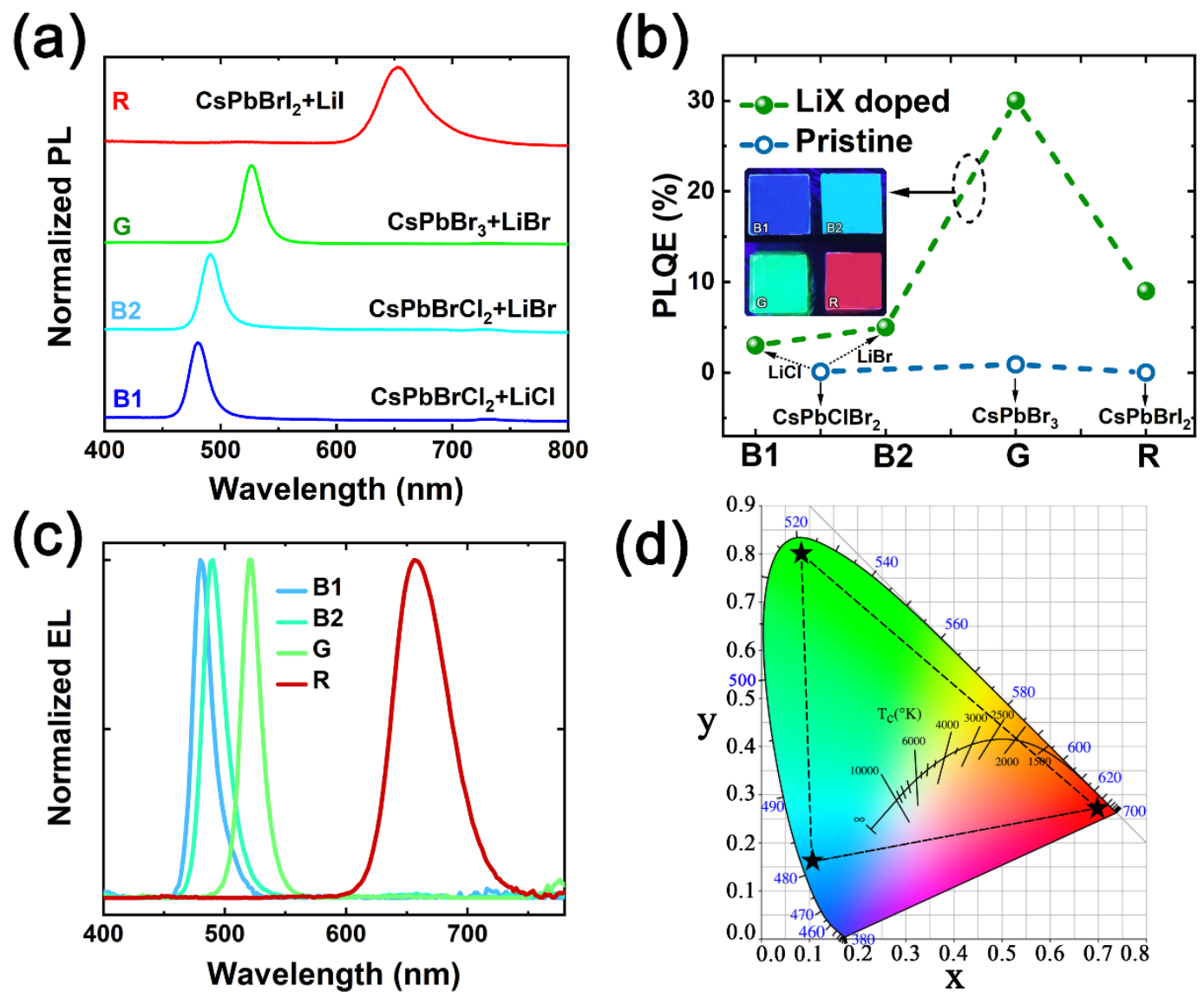

Figure $5 \mathrm{LiX}$ passivation for mixed-halide blue, green and red perovskite films. (a)

PL spectra and (b) PLQE of LiX incorporated blue, green, and red perovskite films. Inset in (b): PL photographs of perovskite films under a $365 \mathrm{~nm}$ UV-lamp illumination; (c) Normalized EL spectra of blue, green and red perovskite LEDs; (d) The corresponding CIE coordination of B1, G, and R LEDs. 
Table 1 Characteristics of electrical output of PeLEDs based on reference, $10 \% \mathrm{LiBr}$, and $20 \% \mathrm{LiBr}$ perovskite films

\begin{tabular}{lccccc}
\hline Perovskite & $\mathrm{V}_{\mathrm{T}}(\mathrm{V})$ & $\mathrm{CE}_{\max }$ & $\mathrm{PE} @ \mathrm{CE}_{\max }$ & $\mathrm{EQE}_{\max }$ & \\
\hline Reference & 3.0 & 9.68 & 5.07 & 2.83 & 26230 \\
$10 \% \mathrm{LiBr}$ & 2.9 & 27.1 & 21.3 & 7.92 & 35400 \\
$20 \% \mathrm{LiBr}$ & 2.4 & 56.9 & 44.6 & 16.2 & 50278 \\
\hline
\end{tabular}




\section{The table of contents entry}

An all-inorganic perovskite light emitting diode with both high efficiency and longterm stability was fabricated through passivation effects of lithium halide. The lithium halide can effectively passivate the surface defects which leads to high ratio of radiative recombination.

High-Performance Perovskite Light-Emitting Diode with Enhanced Operational Stability Using Lithium Halide Passivation

Tian Wu, Junnan Li, Yatao Zou, Hao Xu, Kaichuan Wen, Shanshan Wan, Sai Bai*, Tao Song*, John A. McLeod*, Steffen Duhm, Feng Gao, Baoquan Sun*

\section{TOC Figure}

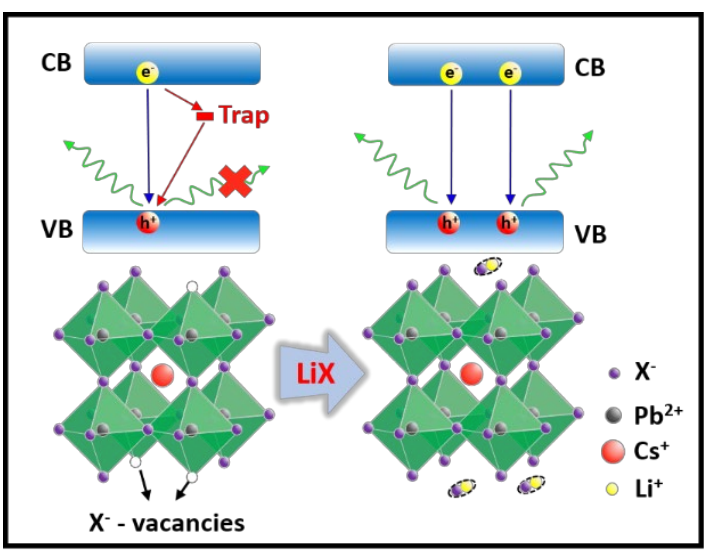

